\title{
EYACULACIÓN ANTERÓGRADA POSTERIOR A LINFADENECTOMÍA LUMBOAÓRTICA LAPAROSCÓPICA MODIFICADA
}

\author{
Gonzalo Valero Fuentealba.
}

Unidad de Laparoscopía. Servicio de Urología. Hospital Regional Rancagua y Hospital Clínico FUSAT.

Rancagua. Chile.

\begin{abstract}
Resumen.- OBJETIVO: El cáncer testicular afecta a pacientes jóvenes y fértiles. La mitad de ellos corresponden a tumores no seminomas. El manejo de este cáncer en Etapa I contempla: observación cuidadosa, quimioterapia y disección ganglionar retroperitoneal (LALA). En el pasado, la LALA clásica producía una alta tasa de aneyaculación con el compromiso posterior de la fertilidad. Actualmente con la técnica modificada se logra una eyaculación anterograda cercana al 90\%. El objetivo de este trabajo es mostrar la conservación de la eyaculación anterograda y algunos aspectos quirúrgicos y oncológicos de la LALA laparoscópica modificada.
\end{abstract}

MÉTODO: Desde enero de 2003 hasta julio de 2006, 24 pacientes portadores de un tumor testicular no seminoma fueron sometidos a LALA laparoscópica modificada. A las seis semanas post cirugía se le preguntó al paciente por la conservación de la eyaculación anterógrada. Se analizan también variables quirúrgicas y oncológicas de la serie.

RESULTADOS: Todas las cirugías fueron completadas en forma exitosa mediante laparoscopía. Edad promedio 29,8 años (15-50). La eyaculación anterógrada se conservó en $91,6 \%(22 / 24)$ de los pacientes. El tiempo quirúrgico promedio fue de 122 minutos (90215). Tiempo de hospitalización promedio 1,7 días (1-4). El promedio de ganglios resecados fue de 13 (5-22). Compromiso ganglionar metastático se encontró en 29\% (7/24) de pacientes. No hubo complicaciones quirúrgicas.

CONCLUSIONES: La vía laparoscópica es tan efectiva como la técnica clásica abierta para mantener la eyaculación anterógrada y la fertilidad posterior. Tiene además una baja morbilidad, las ventajas del acceso mínimamente invasivo y los resultados oncológicos son similares a la vía clásica. Por estos motivos el abordaje laparoscópico debiera ser la técnica de elección para los pacientes en que se decide realizar un estadiaje quirúrgico.

Palabras clave: Linfadenectomía lumboaórtica. Laparoscopía. Eyaculación anterógrada.

Summary.- OBJECTIVES: Testicular cancer affects young and fertile patients. Half of them are non seminomatous tumors. The management of this cancer in stage I includes: watchful waiting, chemotherapy and retroperitoneal lymph node dissection (RPLND). In the past, 
classic RPLND produced a high rate of anejaculation with the subsequent compromise to fertility. Currently, antegrade ejaculation is achieved in almost $90 \%$ of the cases with the modified technique. The objective of this work is to show the conservation of antegrade ejaculation and some surgical anal cortical features of the modified laparoscopic RPLND.

METHODS: From January 2003 to July 200624 patients with non-seminomatous testicular tumors underwent modified laparoscopic RPLND. Patients were asked about preservation of antegrade ejaculation six weeks after surgery. We also analyze surgical and oncological variables in the series.

RESULTS: All surgeries where successfully completed laparoscopically. Mean age 29.8 years (15-50). Antegrade ejaculation was preserved in $91.6 \%(22 / 24)$ of the patients. Mean surgical time was 122 minutes (90-215). Mean hospital stay was 1.7 days (1-4). Mean number of lymph nodes excised was 13 (5-22). Metastatic lymph node involvement was found in 29\% of the patients (7/24). There were no surgical complications.

CONCLUSIONS: Laparoscopic approach is as effective as the classic open technique to maintain antegrade ejaculation and subsequent fertility. Additionally, it has low morbidity, the advantages of a minimally invasive approach and oncological outcomes are similar to the classic approach. For these reasons the laparoscopic approach should be the technique of choice for patients in who decision to undergo surgical staging is taken.

Keywords: Retroperitoneal lymph node dissection. Laparoscopy. Antegrade ejaculation.

\section{INTRODUCCIÓN}

Los tumores de testículo son poco frecuentes y constituyen el principal cáncer en hombres entre los 15 y 35 años de edad. El cáncer testicular se ha transformado en una de las principales enfermedades malignas con un alto nivel de curación. Lo anterior se explica por métodos diagnósticos más efectivos, mejores drogas quimioterápicas, la existencia de marcadores tumorales que optimizan el seguimiento y control y las modificaciones quirúrgicas en la etapificación que han reducido la morbimortalidad del procedimiento.

Aproximadamente la mitad de los tumores de células germinales corresponden a la variedad histológica no seminoma. En este grupo de tumores que se encuentran en Etapa I (TI-3, NO, MO) las op- ciones de manejo son básicamente tres: observación cuidadosa, quimioterapia y linfadenectomía lumboaórtica (LALA).

El uso racional de la LALA radica en que los ganglios lumboaórticos son los principales y únicas vías de propagación extragonadal y por lo tanto la cirugía es capaz de ofrecer tratamiento curativo a la gran mayoría de pacientes con tumores N1-N2. Por otro lado, con los métodos diagnósticos actuales, aproximadamente un $30 \%$ de los pacientes están subetapificados y pueden tener compromiso ganglionar metastático microscópico que no es posible identificar con los métodos de imágenes actuales.

La LALA es una gran cirugía con una morbilidad de $5-25 \%$ y una mortalidad menor a $1 \%$. Las principales complicaciones son íleo, neumonía, atelectasias y linfocele. A lo anterior debe agregarse la gran cicatriz que produce un impacto estético no despreciable, especialmente en este grupo de pacientes jóvenes.

En 1990 Donohue, describe su técnica de disección modificada logrando tasas de eyaculación anterógrada cercanas al $100 \%$ con fertilidad posterior de $75 \%(1)$.

Con el advenimiento de la cirugía de invasión mínima, a mediados de los años noventa se describieron las primeras disecciones ganglionares laparoscópicas con resultados bastante similares a la cirugía abierta y con los beneficios ampliamente aceptados y reconocidos del abordaje laparoscópico (2-4).

El objetivo de este trabajo es mostrar la eyaculación anterógrada que se logra después de LALA laparoscópica modificada y mostrar resultados oncológicos y quirúrgicos.

\section{MATERIAL Y MÉTODO}

Desde enero de 2003 hasta junio de 2006, 24 pacientes con un tumor testicular no seminoma en etapa I fueron sometidos a LALA laparoscópica modificada siguiendo el patrón de disección propuesto por Donohue.

A las seis semanas post cirugía, mediante entrevista personal se preguntó en forma dirigida acerca de la eyaculación anterógrada.

Con todos los pacientes se discutieron las alternativas terapéuticas y después de obtener un consentimiento informado se planeó la LALA. 


\section{Técnica Quirúrgica}

Todos los pacientes fueron operados con anestesia general. Se utilizó profilaxis antibiótica con cefalosporinas y medias elásticas para prevención de trombosis venosa de extremidades inferiores.

Estando el paciente en posición de lumbotomía, se realizó neumoperitoneo con aguja de Verres hasta $15 \mathrm{~mm}$ de $\mathrm{Hg}$ a nivel del hipocondrio, dos cms por debajo del reborde costal. Se usaron cuatro trócares: $10 \mathrm{~mm}$ paraumbilical (óptica de 0 grado), $10 \mathrm{~mm}$ en fosa ilíaca, $5 \mathrm{~mm}$ en hipocondrio y $5 \mathrm{~mm}$ en flanco para retracción ureteral (Figura 1). Para la disección se utilizó coagulación monopolar y el sistema bipolar Ligasure ${ }^{\circledR}$ (Valleylab, Colorado, USA).

Inicialmente se comprueba la ausencia de lesiones intrabdominales y se liberan las adherencias que pudieran existir. Se continúa la disección con el rechazo del colon hacia la línea media mediante una incisión por la línea de Toldt y del ligamento reno-cólico, con lo cual se logra exponer el músculo psoas, el uréter, los grandes vasos retroperitoneales y el hilio renal.

Se realiza disección y exéresis del cordón espermático desde el anillo inguinal profundo hasta la desembocadura en la vena renal o cava. Con disector tipo hook, hacemos la disección de todo el tejido ganglionar con especial cuidado de las venas lumbares.

El tejido correspondiente al cordón espermático y ganglios lumbo-aórticos son introducidos en bolsa plástica intrabdominal y luego retirados en forma íntegra a través de uno de los orificios de los trócares.

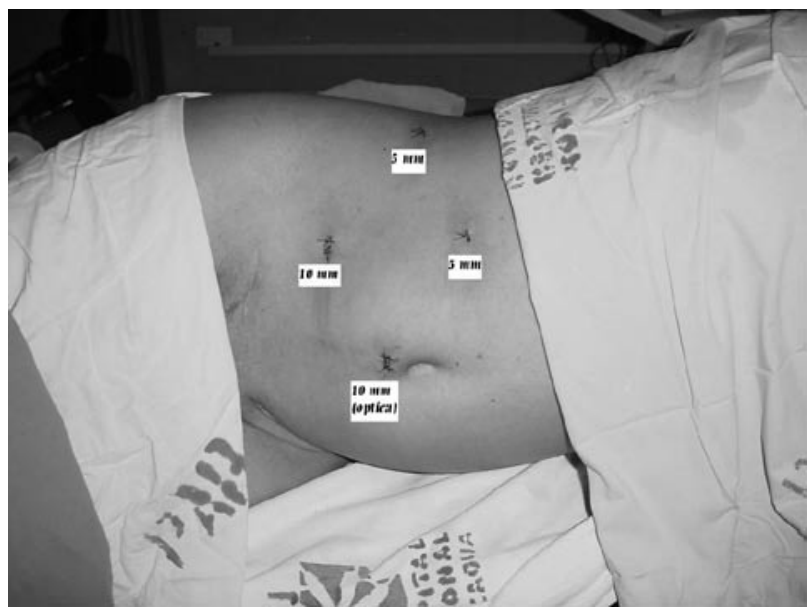

FIGURA 1. Posición del paciente y de los trócares.

\section{RESULTADOS}

Todas las cirugías fueron completadas en forma exitosa mediante la vía laparoscópica. La edad promedio de los pacientes fue de 29,8 años (15-50). La eyaculación anterógrada se conservó en $91,6 \%$ $(22 / 24)$ de los pacientes.

El tiempo quirúrgico promedio fue de $122 \mathrm{mi}-$ nutos (90-215). El tiempo de hospitalización promedio fue de 1,7 días (1-4). El promedio de ganglios resecados fue de 13 (5-22). Se confirmó compromiso ganglionar metastático en $29 \%(7 / 24)$ de pacientes los cuales fueron sometidos a quimioterapia según protocolo. No hubo complicaciones quirúrgicas mayores.

\section{DISCUSIÓN}

En la actualidad la vía laparoscópica constituye un pilar de apoyo fundamental para la práctica de la urología moderna. Es así como existen indicaciones cada vez mayores del acceso laparoscópico con resultados tremendamente satisfactorios y comparables a la vía clásica.

La linfadenectomía lumboaórtica tampoco ha sido la excepción, lo cual queda demostrado con los resultados obtenidos de esta serie. A pesar de que se trata de una cirugía compleja se han obtenido resultados quirúrgicos y oncológicos satisfactorios y comparables a otras series (5-7).

La tasa de eyaculación anterógrada reportada por otros estudios es cercana al 100\% (6-8), por lo que el hecho de tener una tasa por sobre el $90 \%$ nos produce mucha satisfacción y gran optimismo con respecto a la fertilidad posterior de nuestros pacientes. Al cierre de la revisión de esta serie había un paciente con embarazo logrado.

Con respecto a la calidad de vida percibida por los pacientes sometidos a cirugía laparoscópica, Poulakis y cols muestran en un estudio comparativo entre ambas vías de acceso que en la primera los pacientes tienen menor tiempo de hospitalización, mejor calidad de vida objetivada mediante cuestionarios validados y una más rápida recuperación y retorno a la vida laboral (9). Aunque evaluar este punto no ha sido el fundamento de este estudio, es posible afirmar que todos los pacientes han logrado reincorporarse a la vida laboral en forma precoz y un aspecto importante para ellos también ha sido la parte estética.

Un aspecto importante y que cobra cada vez mayor relevancia es el costo de los procedimientos e 
intervenciones médicas. La cirugía laparoscópica en general es más costosa que la vía clásica debido a la prolongación de los tiempos quirúrgicos y al uso de materiales específicos y desechables. Un interesante estudio llevado a cabo por Ogan y cols. mostró que los costos de la vía laparoscópica podían ser reducidos cuando los tiempos de cirugía no excedían las dos horas y el paciente era enviado a su casa antes de 48 horas (10). En la medida en que se realizan mayor cantidad de procedimiento y se logra pasar la curva de aprendizaje los tiempos quirúrgicos así como también el número de complicaciones se va optimizando para lograr reducir los costos de la cirugía laparoscópica. En la serie mostrada el tiempo quirúrgico es de 2 horas y la hospitalización menor a dos días, lo cual sin duda nos ha permitido reducir los costos médicos e incluso generar ahorros si se toma en cuenta que el tiempo de estadía de los pacientes operados por vía abierta no es inferior a 4-5 días.

El seguimiento a largo plazo de los pacientes sometidos a LALA laparoscópica también ha sido satisfactorio según las series reportadas por Bhayani y cols., Steiner y cols. y Valdebenito y cols. Con seguimientos que bordean los 6 años han mostrado tasas de recaídas similares a la cirugía abierta que han sido rescatadas con quimioterapia y una sobrevida global y enfermedad específica de 100\% (11-13).

\section{CONCLUSIÓN}

La LALA laparoscópica modificada logra conservar la eyaculación anterógrada y posee resultados oncológicos similares a la vía clásica con las bondades del abordaje laparoscópico. En la actualidad no cabe duda que debiera ser la vía de elección en pacientes que van a ser sometidos a estadiaje quirúrgico.

\section{BIBLIOGRAFÍA Y LECTURAS RECOMENDADAS (*lectura de interés $y^{* *}$ lectura fundamental)}

**1. DONOHUE, J.P.; FOSTER, R.S.; ROWLAND, R.G. y cols.: "Nerve sparing retroperitoneal lymphadenectomy with preservation of ejaculation". J. Urol., 144: 287, 1990.

2. RASSWEILER, J.J.; SEEMANN, O.; HENKEL, T.O. y cols.: "Laparoscopic retroperitoneal lymph node dissection for nonseminomatous germ cell tumors: Indications and limitations". J. Urol., 156: 1108, 1996.

3. JANETSCHEK, G.;HOBISCH, A.; HOLTL, L. y cols.: "Retroperitoneal lymphadenectomy for clinical stage I nonseminomatous testicular tumor: Laparoscopy versus open surgery and impact of learning curve". J. Urol., 156: 89, 1996.

4. GERBER, G.S.; BISSADA, N.K.; HULBERT, J.C. y cols.: "Laparoscopic retroperitoneal lymphadenectomy: Multi-institutional analysis". J. Urol., 152: 1188, 1994.

5. JANETSCHEK, G.; HOBISCH, A.; REINHARD, P. y cols.: "Retroperitoneal lymph node dissection for clinical satge I nonseminomatous testicular carcinoma: Long term outcome". J. Urol., 163: 1793,

6. NEYER, M.; PESCHEL, R.; AKKAD, T. y cols.: "Long-term results of laparoscopic retroperitoneal lymph-node dissection for clinical stage I nonseminomatous germ-cell testicular cancer". J. Endourol., 21: 180, 2007.

7. NELSON, J.; CHEN, R.; BISHOFF, J. y cols.: "Laparoscopic retroperitoneal lymph node dissection for clinical stage I nonseminomatous germ cell testicular tumor". Urology, 54: 1064, 1999.

**8. CASTILLO, O.; ALVAREZ, J.; VITAGLIANO, G. y cols.: "Linfadenectomía retroperitoneal laparoscópica en cáncer de testículo no seminoma estadio I". Arch. Esp. Urol., 60: 59, 2007.

*9. POULAKIS, V.; SKRIAPAS, K.; DE VRIES, R. y cols.: "Quality of life after laparoscopic and open retroperitoneal lymph node dissection in clinical Stage I nonseminomatous germ cell tumor: A comparison study". Urology, 68: 154, 2006.

*10. OGAN, K.; LOTAN, Y.; KOENEMAN, K. y cols.: "Laparoscopic versus open retroperitoneal lymph node dissection: A cost analysis". J. Urol., 168: 1945, 2002.

*11. BHAYANI, S.; ONG, A.; OH, W. y cols.: "Laparoscopic retroperitoneal lymph node dissection for clinical stage I nonseminomatous germ cell testicular tumor: A long-term update". Urology, 62: 324, 2003.

**12. STEINER, H.; PESCHEL, R.; JANETSCHEK, G. y cols.: "Long-term results of laparoscopic retroperitoneal lymph node dissection: A single-center 10 year experience". Urology, 63: 550, 2004.

*13. VALDEBENITO, J.; MERHE, E.; VALDEBENITO, R. y cols.: "Linfadenectomía retroperitoneal reducida en cáncer testicular de células germinales no seminomatoso estadio clínico I". Arch. Esp. Urol., 60: 245, 2007. 